\title{
Immobilization and microencapsulation
}

\author{
Gauri Aeron and Shiwangi Morya* \\ Department of Biotechnology, S.D. College of Engineering \& technology, Muzaffarnagar-251001, India
}

Received: 09 November, 2017; Accepted: 30 November, 2017; Published: 20 December, 2017

*Corresponding author: Shiwangi Morya, Department of Biotechnology, S.D. College of Engineering \& technology, Muzaffarnagar-251001, India, Tel: +919997917215; E-mail: shiwangimorya97@gmail.com

\begin{abstract}
The utilization of probiotics is continuously increasing due to various benefits conferred on the health of consumers. Probiotic foods are a group of functional foods with growing market shares and large commercial interest. In this context, the viability of the probiotic culture in food products is favored by Microencapsulation, mainly by the properties of protection against adverse environmental conditions and controlled release. The most popular system of immobilization reported is entrapment of cells in a gel matrix of alginate. The beads are formed by this method have a range of $2-3 \mathrm{~mm}$ in diameter. In case of alginate capsule the extrusion method involve the following steps: preparation of hydrocolloid solution, addition of probiotic cells into the above mentioned solution in order to form cell suspension and extrusion of the cell suspension through syringe needle.
\end{abstract}

\section{Introduction}

Probiotics are the living microbes which are when ingested to the human it confer the beneficial effects on the equilibrium and the physiological functions of the human intestinal microflora. Probiotics are defined as "live microbes that gives a health benefits on the host when administered in adequate amounts." i.e. Probiotic are live micro-organism that can be formulated into many different types of products, including foods, drugs, and dietary supplement. Probiotics is a relatively new word that is used to name the bacteria connected with the valuable effects for the humans and animals. The term probiotics is used "for life" and it was defined by an Expert Committee as "live microorganism which upon ingestion in definite numbers exerts health benefits further than inherent general nutrition." FAO/WHO Expert Support believes that general guidelines need to provide to how these microorganism can be tested and proven for safety and potential health benefits when administered to humans.

Lactic Acid Bacteria (LAB) which have been used for food fermentation since the earliest time, can serve a dual function by acting as food fermenting agent and potentially health benefits provider. LAB are GRAS with no pathogenic, or virulence properties have been reported. For the use of LAB as probiotics, there are some required characteristics e.g. low cost, maintaining its viability during the processing and storage, facility of the application in the products, opposition to the physiochemical processing must be considered.

In the recent past, there has been an unexpected increase in probiotics- based health products mainly in the form of fermented dairy products as well as dietary supplements. Today there are more than 70 "Bifidus"-and "Acidophilus"- containing products worldwide, including some fermented dairy products (Shah, 2001).

The utilization of probiotics at a level of $10^{8}-10^{9} \mathrm{cfu} / \mathrm{gm}$ perday is a commonly quoted figure for adequate probiotic consumption, equating to $100 \mathrm{~g}$ of a food product with $10^{6}$ $10^{7} \mathrm{cfu} / \mathrm{g}$ (Kebary, 1996; Lee and Salminen, 1996; Dave and Shah, 1997c). In many different countries, analysis of probiotic products has confirmed that probiotic strains exhibit poor survival in traditional fermented dairy products (Shah, 2000, Lourens-Hattingh and Viljoen, 2001). The probiotic preparations such as tablets, powders etc. may contain lower viable counts. Of the 20 feed supplements studied, viable probiotic counts varied greatly, with 3 products containing no lactobacilli at all (Gilliland, 1981), although the supplements were supposed to contain L. acidophilus. Probiotic survival in products is affected by a range of factors such as $\mathrm{pH}$, post-acidification (during storage) in fermented products, hydrogen peroxide production, oxygen toxicity (oxygen permeation through packaging), storage temperatures, stability in dried or frozen form, poor growth in milk, lack of proteases to break down milk protein to simpler nitrogenous substances and compatibility with traditional starter culture during fermentation (Dave and Shah, 1997a, b, c; Kailasapathy and Rybka, 1997; Shah, 2000). Oxygen plays a major role in the poor survival of probiotic bacteria (Brunner et al., 1993). Provided that probiotic living cells with a physical barrier against adverse external conditions is an approach currently receiving considerable curiosity. For use in bio-technological applications, in the past, microorganisms were immobilized or entrapped in polymer matrices.

\section{Immobilization and microencapsulation}

Entrapment of cells in an alginate gel of sodium alginate is the most common system of immobilization reported. The terms immobilization and encapsulation were used interchangeably in the majority of reported literature. While encapsulation is the process of forming an unremitting varnish around an inner matrix that is utterly contained within the capsule wall as a core of encapsulated material, immobilization refers to the trapping of material within or throughout a matrix. A small percentage of immobilized material may be uncovered at the surface, while this 
is not the case for encapsulated material (King, 1995). Naturally, Encapsulation occurs when bacterial cells grow and produce exopolysaccharides. The entrapped microbial cells within their own secretions that act as a protective/shielding structure or a capsule, falling the permeability of material through the capsule and therefore less exposed to adverse / unfavorable environmental factors. Many lactic acid bacteria synthesize exo-polysaccharides, but they produce inadequate exo-polysaccharides to be able to encapsulate themselves fully (Shah, 2002). Microencapsulation helps to split a core material from its environment until it is released. It protects the unstable core from its environment, thereby improving its stability, extends the core's shelf life and provides a constant and controlled release. The structure build by the micro-encapsulation agent around the core substance is known as the wall. The properties of the wall system are intended to protect the core and to release it at controlled rates under specific conditions while allowing small molecules to pass in and out of the membrane (Franjione and Vasishtha, 1995; Gibbs et al., 1999). The capsules may range from submicron to several millimetres in size and can be of different shapes (Shahidi and Han, 1993; Franjione and Vasishtha, 1995). Contrast to immobilization or entrapment techniques, micro-encapsulation has countless advantages. The microcapsule is composed of a semi permeable, spherical, thin and strong membranous wall. Therefore the bacterial cells are retained within the microcapsules (Jankowski et al., 1997). Moreover, compared to an entrapment matrix, there is no solid or gelled core in the microcapsule and its small diameter helps to reduce mass transfer limitations. The nutrients and metabolites can diffuse through the semi permeable membrane easily. The membrane serves as a barrier to cell release and minimizes contamination. The encapsulated core material is unconfined by several mechanisms such as mechanical rupture of the cell wall, dissolution of the wall, thaw out of the wall and diffusion through the wall.

\section{Effect of using immobilized LAB}

There are several benefits of using immobilized LAB with respect to free bacteria -

- The concentration of cell in immobilization is higher than free suspension.

- Immobilization provides cells to reuse and eliminates the costly processes of cell recovery and cell recovery.

- At high dilution rate problems like cell washout is eliminated by immobilization.

- Combination of high cell concentration and high flow rate allows high volumetric productivity.

- Favorable micro environmental condition.

- Improve genetic stability.

- Protect against shear damage.

\section{Importance of oxygen on viability of probiotics}

Market surveys on the viability of L. acidophilus and bifidobacteria in probiotic yoghurts vary in their findings with some studies reporting low counts of these bacteria (Iwana et al., 1993; Shah et al., 1995; Rybka and Fleet, 1997; Anon, 1999; Shah et al., 2000) while others citing satisfactory viability (Lourens et al., 2000; Shin et al., 2000). Variable counts have been reported elsewhere (Pacher and Kneifel, 1996; Dave and Shah, 1997c; Micanel et al., 1997; Vinderola and Reinheimer, 1999; Vinderola et al., 2000). Several factors are reported to influence the survival of L. acidophilus and bifidobacteria in yoghurts. These include acid and hydrogen peroxide produced by yoghurt bacteria, type of strain, culture conditions, and concentrations of lactic and acetic acids, whey proteins and interaction of the probiotic species with the yoghurt starters (Kailasapathy and Supraidi, 1996; Godward et al., 2000; Shah, 2000; Vinderola et al., 2000). Among these, the oxygen content in the product and oxygen permeation through the package is considered most significant in reducing the viability of L. acidophilus and bifidobacteria in fermented milk products (Klaver et al., 1993; Dave and Shah, 1997c).

\section{Oxygen toxicity of probiotic bacteria in fermented milks, particularly yoghurts}

Organisms inhabiting the human gut are generally anaerobic or microaerophilic and therefore lack effective oxygen scavenging cellular mechanisms such as catalyses. Hence, exposure to oxygen in these bacteria causes toxic oxygenic metabolites to accumulate in the cell leading to cell death from oxidative damage. This lethal effect of oxygen is termed as oxygen toxicity. Both L. acidophilus and Bifidobacterium spp. are derived from the intestine and are classified as microaerophilic and strictly anaerobic respectively. Dairy foods such as yoghurt contain high levels of oxygen, which is incorporated during the various homogenization, mixing and agitation steps of yoghurt manufacture. Additionally, oxygen diffuses through the packaging material during the shelf life storage (Ishibashi and Shimamura, 1993; Miller et al., 2002). The resulting oxygen environment in yoghurts or fermented milks is thought to induce cell death and lead to poor viability of L. acidophilus and Bifidobacterium spp. (Brunner et al., 1993a; Brunner et al., 1993b; Klaver et al., 1993). Although bifidobacteria are considered more susceptible to oxygen than L. acidophilus due to their anaerobic nature, the oxygen susceptibility of bifidobacteria could however be strain dependent. Dave and Shah (1997b) found that bifidobacteria survived well over a 35 day period in yoghurt, regardless of the oxygen content and redox potential of the yoghurt. Miller et al. (2002) too found better survival of bifidobacteria compared to L. acidophilus even as the dissolved oxygen of the yoghurt was seen to rise steadily over the shelf life. While counts of bifidobacteria remained above the recommended $106 \mathrm{cfu} / \mathrm{g}$ throughout the shelf life of the yoghurt, L. acidophilus counts were found to decrease below $103 \mathrm{cfu} / \mathrm{g}$ by the third week of storage. The strain dependent phenomenon of oxygen sensitivity was further demonstrated by Meile et al. (1997) who were able to isolate a moderately oxygen tolerant species of Bifidobacterium, B. lactis sp. nov. from fermented milk. 
Techniques to protect $L$. acidophilus and Bifidobacterium spp. from oxygen toxicity in yoghurts

\section{Use of acorbate and L-cysteine as oxygen scavengers in yoghurts}

The property of ascorbic acid and L-cysteine to act as oxygen scavengers and maintain low redox potential was evaluated by Collins and Hall (1984) and by Dave and Shah (1997a; 1997b) for maintaining conditions suitable for the extended viability of probiotic bacteria in yoghurts. Although there was a reduction in the oxygen content and redox potential of yoghurt, together with an improvement in the counts of L. acidophilus and Bifidobacterium spp., the incorporation of ascorbic acid in yoghurts can however reduce the amount of oxygen required for the activities of $S$. thermophilus, an aerobic organism used as a starter culture in the manufacture of yoghurt. Cysteine too was found to suppress the growth of the yoghurt starter cultures, $S$. thermophilus and L. delbrueckii ssp. bulgaricus. This can have a detrimental effect on the textural and nutritional qualities of yoghurt and this technique may hence be undesirable in the industrial manufacture of yoghurt.

\section{Use of special high-oxygen consuming strains}

To protect probiotic bacteria, especially bifidobacteria from oxygen in yoghurt, the incorporation of a high-oxygen consuming strain of Streptococcus thermophilus has been suggested (Lourens-Hattingh and Viljoen, 2001). S. thermophilus, which relies heavily on oxygen for its metabolic activities can act as an effective oxygen scavenger by its consumption of the dissolved oxygen in the yoghurt. This can therefore help to reduce oxygen exposure to bifidobacteria (Ishibashi and Shimamura, 1993). This technique however suffers from the drawback that fast acidifying strains of $S$. thermophilus used commercially, can lead to a rapid accumulation of acid in the growth medium. As both L. acidophilus and bifidobacteria are sensitive to high acidity, this can have a negative impact on the viability of probiotic bacteria. Moreover, this method is useful in providing protection against oxygen toxicity only during the stages of yoghurt manufacture. It does little to protect the probiotic bacteria from subsequent oxygen ingress into yoghurt through the packaging material.

\section{Microencapsulation}

Micro-encapsulation of probiotic bacteria is a process in which cells are retained within an encapsulating membrane to reduce cell injury or cell loss (Shah, 2000; Kailasapathy, 2002). The physical retention of cells in the encapsulating matrix can facilitate the separation of cells from direct exposure to the adverse factors such as oxygen while at the same time allow the diffusion of nutrients in and out of the matrix to support the viability of the cells. Microencapsulation has also been applied to increase the survival of probiotic bacteria in yoghurt and other dairy products by protecting probiotic bacteria from lyophilization and rehydration, and acidity in the product (Shue et al., 1993; Dinakar and Mistry, 1994; Kim et al., 1996; Kebary et al., 1998; Adhikari et al., 2000; Shah and Ravula, 2000; Sultana et al., 2000). Little is known about the protective effect of microencapsulation from oxygen toxicity. Talwalkar and Kailaspathy (2003a) evaluated the protective effect of microencapsulation against oxygen toxicity in L. acidophilus and Bifidobacterium spp. in both culture broths as well as in prepared yoghurt. Cells were encapsulated in calcium alginate and grown in the presence of oxygen. When tested in optimum conditions of culture broth and temperature $\left(37^{\circ} \mathrm{C}\right)$, encapsulated cell counts were one log higher than their free cell counterparts. Mixed results were however observed when microencapsulation was tested in yoghurt stored at $6^{\circ} \mathrm{C}$. Microencapsulation was found to significantly increase viability in six strains while no significant difference was observed between encapsulated cell counts and free cells counts in the other six strains. Preliminary evidence therefore suggests that although microencapsulation can offer protection to probiotic bacteria against oxygen toxicity in broth culture, further optimization studies are needed before its application in yoghurt.

\section{Packaging material}

The oxygen permeability of the packaging material used currently for probiotic yoghurts is considered a key factor in the high levels of oxygen present in yoghurt. It is well known that packaging materials such as polyethylene and polystyrene are gas permeable and allow the diffusion of oxygen into yoghurt during storage (Ishibashi and Shimamura, 1993). The exclusion of oxygen during the manufacturing process can be costly. Few current packaging techniques are capable of preventing oxygen permeation. Dave and Shah (1997c) found improved survival of $L$. acidophilus over a 35-day period in yoghurts that were packaged in glass bottles as compared to when the yoghurt was packaged in plastic cups. The oxygen content in yoghurts stored in plastic cups increased due to the permeation of oxygen whereas the yoghurts contained in the glass bottles retained a low oxygen environment. This led to the suggestion that to prevent oxygen toxicity in probiotic bacteria, yoghurts be packed in glass containers. Although effective, glass jars are however neither convenient nor practical owing to their high cost and handling hazards.

\section{Biochemistry of the oxidative response}

Aerobic bacteria derive their energy primarily through oxidative phosphorylation, involving the electron transport chain, which is composed of a chain of carriers capable of undergoing reversible oxidation and reduction. Anaerobic bacteria including lactic acid bacteria such as L. acidophilus and Bifidobacterium spp. cannot synthesize cytochromes and other heme containing enzymes vital to the electron transport chain. They are thus unable to synthesize ATP by respiratory means and have to depend strictly on a fermentative mode of metabolism. Due to the lack of participation of an external electron acceptor (oxygen in aerobic bacteria) in anaerobes, the organic substrate undergoes a balanced series of oxidative and reductive reactions mediated by pyridine nucleotides such as NADH. As the energy in anaerobes is derived mainly through substrate level phosphorylation, the regeneration of NAD+ from NADH assumes critical importance. The simplest way to oxidize NADH is by the reduction of molecular oxygen (02) via the activity of NADH oxidase. Coincidentally, 
possession of a NADH oxidase appears to be a universal property of lactic acid bacteria (Condon, 1987). Generally, the NADH oxidizing reactions in lactic acid bacteria including L. acidophilus and Bifidobacterium spp. catalyze the transfer of one, two or four electrons to the dioxygen molecule (Figure 1).

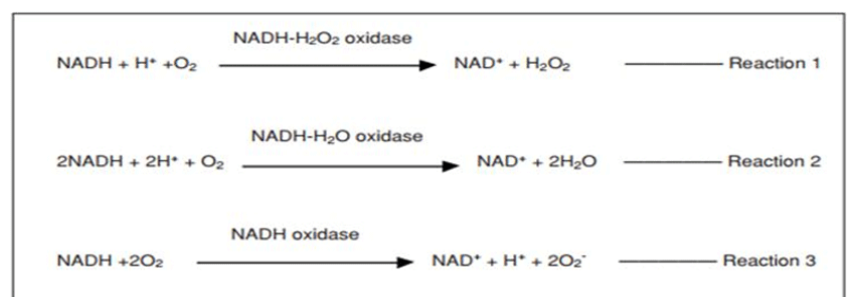

Figure 1: Reactions of NADH oxidase with oxygen in Lab (Condon, 1987)

\section{Conclusion}

Probiotic foods are a collection of functional foods with growing market shares and large commercial interest. Probiotics exert their benefit by way of numerous mechanisms: they prevent colonization, cellular adhesion and invasion by pathogenic organism, they have direct antimicrobial activity and they modulate the immune response. The present research will show the clinical efficiency of probiotics in their utilization for the anticipation of symptoms of lactose intolerance in teenagers, treatment of diarrhea, attenuation of antibiotic associated gastrointestinal side effects and the prevention and treatment of allergic symptoms i.e. the current research will provide a way to rectify all the above mention diseases.

\section{References}

1. Burey P, Bhandari BR, Howes T, Gidley MJ. Hydrocolloid gel particles, formation, characterization, and application. Crit Rev Food Sci Nutr. 2008;48(5):361-377. Doi: 10.1080/10408390701347801.

2. J.Burgain, C Gaiani, M LinderJ Scher. Encapsulation of probiotic living cells: From laboratory scale to industrial applications. Journal of Food Engineering. 2011;104(4):467-483 Doi: org/10.1016/j. jfoodeng.2010.12.031

3. BURGESS, D.J.; HICKEY, A.J. Microsphere technology and applications. Encyclopedia of Pharmaceutical technology. 1994;10:1-29.

4. Fabiane PicininDe Castro-Cislaghi, Carina Dos Reis ESilva, Carlise BeddinFritzen-Freire, Juliana GoulartLorenz, Ernani S.Sant'Anna. Bifi dobacterium Bb-12 microencapsulated by spray drying with whey: Survival under simulated gastrointestinal conditions, tolerance to $\mathrm{NaCl}$, and viability during storage. Journal of Food Engineering. 2012;113(2):186-193. Doi: 10.1016/j.jfoodeng.2012.06.006

5. Claude P Champagne, Patrick Fustier. Microencapsulation for the improved delivery of bioactive compounds into foods. Current Opinion in Biotechnology. 2007;18(2):184-90. Doi: 10.1016/j. copbio.2007.03.001.

6. Kun-Nan Chen, Ming-Ju Chen, Je-Ruel Liu, Chin-Wen Lin, HsinYi Chiu. Optimization of Incorporated Prebiotics as Coating Materials for Probiotic Microencapsulation. Journal of Food Science. 2005;70(5):260-266. Doi: 10.1111/j.1365-2621.2005.tb09981.x.

7. Chandramouli V, Kailasapathy K, Peiris P, Jones M. An improved method of microencapsulation and its evaluation to protect Lactobacillus spp. in simulated gastric conditions. Journal of Microbiological Methods.2004;56(1):27-35.

8. Chávarri M, Marañón I, Ares R, Ibáñez FC, Marzo F, Villarán Mdel C. Microencapsulation of a probiotic and prebiotic in alginate-chitosan capsules improves survival in simulated gastro-intestinal conditions. Int J Food Microbiol. 2010; 142(1-2):185-189. Doi: 10.1016/j. ijfoodmicro.2010.06.022.

9. Kun-Nan Chen, Ming-Ju Chen, Je-Ruel Liu, Chin-Wen Lin, HsinYi Chiu. Optimization of Incorporated Prebiotics as Coating Materials for Probiotic Microencapsulation. Journal of Food Science. 2005;70(5):260-266. Doi: 10.1111/j.1365-2621.2005.tb09981.x. 